\title{
The Effects of Vascular Endothelial Growth Factor on Capsule Formation around Silicone Implant in Rats
}

\author{
Da Han Kim¹, You Seong Song ${ }^{2}$, \\ Seung Yong Song ${ }^{3}$, \\ Chung Hun Kim² \\ ${ }^{1}$ Yonsei E1 Plastic Surgery Clinic, Anyang; \\ ${ }^{2}$ Department of Plastic and \\ Reconstructive Surgery, CHA Bundang \\ Medical Center, CHA University School of \\ Medicine, Seongnam; ${ }^{3}$ Department of \\ Plastic and Reconstructive Surgery, \\ Yonsei University College of Medicine, \\ Seoul, Korea
}

\begin{abstract}
Background The purpose of this study was to examine the effects of a single administration of vascular endothelial growth factor (VEGF) in promoting the angiogenesis and thereby reducing the formation of capsular contracture.

Methods We treated 24 female Sprague-Dawley rats with (1) $5 \mathrm{mM}$ Tris Buffer and 150 $\mathrm{mM} \mathrm{NaCl} 0.1 \mathrm{cc}$, (2) VEGF $15 \mu \mathrm{g} / 0.1 \mathrm{cc}$, (3) VEGF $150 \mu \mathrm{g} / 0.1 \mathrm{cc}$ during placement of the implant, or (4) VEGF $150 \mu \mathrm{g} / 0.1 \mathrm{cc}$ and VEGF $300 \mu \mathrm{g} / 0.2 \mathrm{cc}$. We histopathologically measured the thickness of the capsule and the number of blood vessels.

Results All experimental groups had a significant difference in the thickness of the capsule compared to the control group $(\mathrm{P}<0.001)$. There was no significant difference between experimental group 2 and experimental group 3. The number of blood vessels formed around the capsule was significantly greater in all the experimental groups compared with the control group $(P<0.05)$. There was no significant difference between the experimental groups. There was a significant negative correlation between the thickness of the capsule and the number of blood vessels (Spearman's correlation coefficient, 0.2732; $\mathrm{P}<0.0001$ ).

Conclusions A single administration of VEGF reduced formation of the capsule and increased the vascularity around the implant, supporting the hypothesis that prevention of tissue ischemia can be a treatment strategy for capsular contracture.
\end{abstract}

Keywords Breast implants, Contracture, Rats, Vascular endothelial growth factor

this article was reported.

\section{INTRODUCTION}

Silicone breast implants have been used for breast augmentation and reconstructive surgery for the past 40 years. Capsular contracture is a relatively common complication (a capsule that forms around the breast implant, thereby causing a deformity and pain), and poses challenging problems for surgeons [1]. Clinically, patients with severe capsular contracture, such as those with capsular contracture grade III or IV (Baker's classification), experience pain or

Received: Aug 29, 2016 Revised: Sep 17, 2016 Accepted: Sep 26, 2016 Correspondence: Chung Hun Kim Department of Plastic and Reconstructive Surgery, CHA Bundang Medical Center, CHA University School of Medicine, 59, Yatap-ro, Bundang-gu, Seongnam 13496, Korea. E-mail:chkimps@cha.ac.kr

Copyright () 2016 The Korean Society for Aesthetic Plastic Surgery.

This is an Open Access article distributed under the terms of the Creative Commons Attribution Non-Commercial License (http://creativecommons.org/licenses/by-nc/4.0/) which permits unrestricted non-commercial use, distribution, and reproduction in any medium, provided the original work is properly cited. www.e-aaps.org tenderness, which may eventually lead to poor functional and aesthetic outcomes [2]. Severe capsular contractures have been corrected by capsulotomy or capsulectomy; other treatment methods are to change the location of the breast implants or replace them with other products that have a different surface property $[3,4]$. There are no established methods to prevent the occurrence of capsular contracture.

The exact pathophysiology of capsular contracture remains unclear, but previous studies have suggested that it is associated with chronic inflammatory factors, such as a foreign body reaction to the implant, the surface property of the implant, site of implant placement, postoperative bacterial infections, hematoma, or seroma $[5,6]$. Studies have examined whether inhibiting inflammatory events that cause a fibrosis is effective in preventing the capsular contracture, demonstrating that anti-inflammatory agents (e.g., calcium channel blockers), angiotensin-converting enzyme inhibitors, and leukotriene receptor antagonists (e.g., zafirlukast and montelukast) were effective in reducing the degree of capsular contracture [7-11].

Studies have reported, however, that ischemic changes may lead 
to tissue fibrosis $[12,13]$. We performed a preliminary animal experiment, which showed that human embryonic stem cell (hESC)induced endothelial progenitor cell (EPC) culture medium (CM) promoted angiogenesis and reduced formation of the peri-implant capsule [14].

We hypothesized that the vascular endothelial growth factor (VEGF) would be effective in promoting angiogenesis and thus reducing the formation of capsular contracture. We demonstrate the effects of VEGF in an animal model using Sprague-Dawley (SD) rats in this study.

\section{METHODS}

\section{Experimental animals and setting}

Prior to the experimental procedure, the study protocol was approved by the Institutional Animal Care and Use Committee of our medical institution (IACUC130001).

We used 24 female SD rats, weighing average 250 to $300 \mathrm{~g}$, housed in individual cages at a temperature of $21 \pm 2^{\circ} \mathrm{C}$ under a 12-hour light/12-hour dark cycle, and provided with food and water ad libitum. To increase the accuracy of experimental data, we adapted the SD rats to the laboratory environment for one week before beginning the experiment.

A silicone implant with a similar shape to a human breast prosthesis was custom ordered and prepared as a silicone lump with a smooth surface. The implant had a diameter of $18 \mathrm{~mm}$ and a maxi-

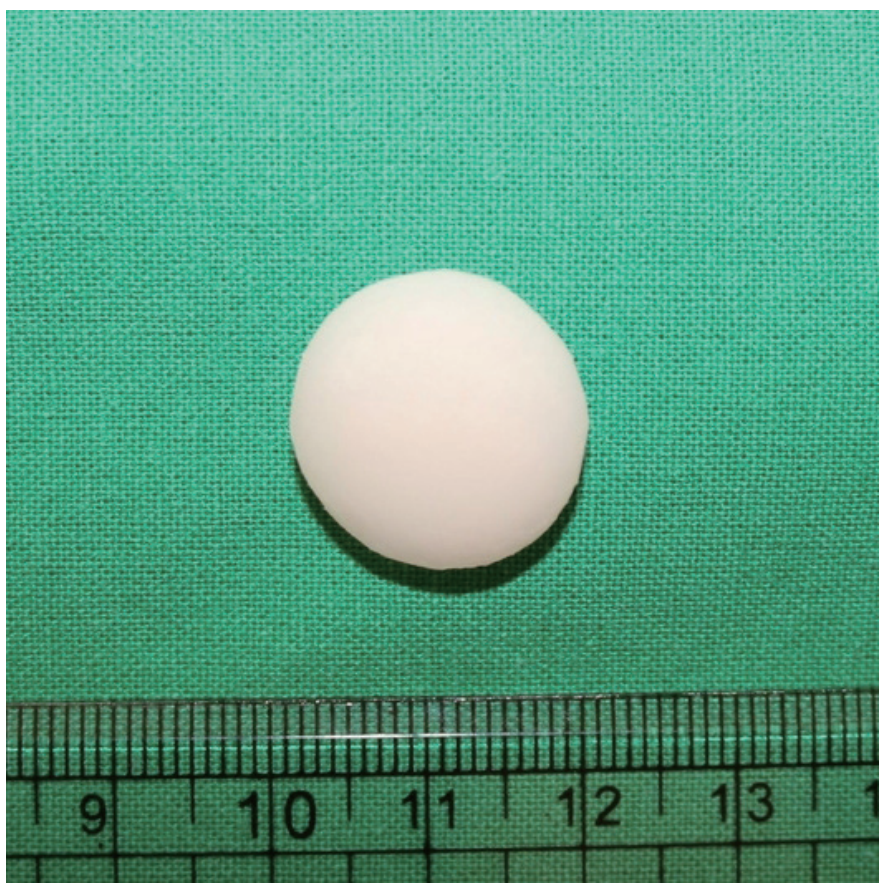

Fig. 1. Silicone implant used for the experiment. A silicone lump with a smooth surface was used as a custom silicone implant; it had a diameter of $18 \mathrm{~mm}$ and a maximal thickness of $7 \mathrm{~mm}$. mal thickness at the core of average $7 \mathrm{~mm}$ (Fig. 1). The prepared silicone implant was sterilized using ethylene oxide for the current experimental procedure.

In the laboratory procedure, we used human recombinant VEGF (CHA-VEGF ${ }^{\circledR}$; Cha Bio \& Diostech, Seoul, Korea).

\section{Animal study}

The SD rats were given tiletamine/zolazepam (Zoletil ${ }^{\circledR}$; Zoetis Animal Health, Lincoln, NE, USA; $60 \mathrm{mg} / \mathrm{kg}$ ) and xylazine (Rompun ${ }^{\circledR}$; Bayer, Leverkusen, Germany; $18.6 \mathrm{mg} / \mathrm{kg}$ ) intra-abdominally for anesthesia. Rats were aseptically disinfected with $10 \%$ povidoneiodine solution in a prone position. An incision $(2 \mathrm{~cm})$ was made along the median line of the vertebral spines. Through a lateral dissection, we created a subcutaneous pocket underneath the panniculus carnosus muscle, where we placed the silicone implant. The skin incision window was sutured using Nylon 4-0 sutures (Blue Nylon $^{\circledR}$, Busan, Korea), and followed by an intramuscular injection of gentamicin (0.05 mL/kg; Fig. 2).

\section{Dosing rationale}

The 24 SD rats were divided into four groups (1 control group and 3 experimental groups):

(1) The control group $(n=7)$ : The SD rats received an intradermal injection of $5 \mathrm{mM}$ Tris Buffer and $150 \mathrm{mM} \mathrm{NaCl}$ at a dose of $0.1 \mathrm{cc}$ to the region adjacent to the implant.

(2) The experimental 1 group $(n=7)$ : The SD rats received an

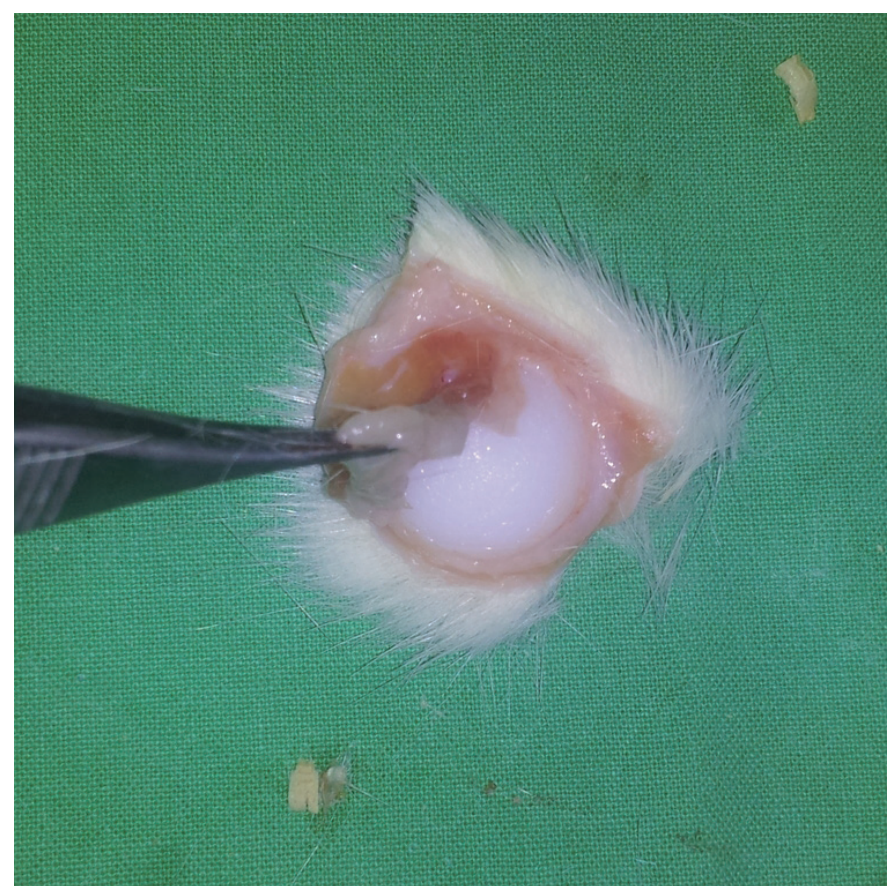

Fig. 2. Gross findings of the tissue sample. On gross examination of the extracted tissue samples, a prosthesis was surrounded by a fibrous capsule with a well-defined margin from the adjacent tissue. On palpation, the capsule had a firm, smooth consistency. 
intradermal injection of VEGF at a dose of $15 \mu \mathrm{g} / 0.1 \mathrm{cc}$ to the adjacent region of the implant during placement.

(3) The experimental group $2(n=7)$ : The $S D$ rats received an intradermal injection of VEGF at a dose of $150 \mu \mathrm{g} / 0.1 \mathrm{cc}$ during placement of the implant.

(4) The experimental group $3(n=3)$ : The SD rats received an intradermal injection of VEGF at a dose of $150 \mu \mathrm{g} / 0.1 \mathrm{cc}$ during placement of the implant, followed by an intradermal injection of VEGF at a dose of $300 \mu \mathrm{g} / 0.2 \mathrm{cc}$ on postoperative day 5 .

We analyzed the dose-response relationship in experimental group 2 compared with experimental group 1 . We also analyzed the effects of multiple dosing in experimental group 3 compared with experimental group 2.

\section{Histopathological examinations Histopathological examinations}

On postoperative week 8 , the SD rats were sacrificed. The silicone implant and its adjacent tissue were dissected in one piece. Following the removal of a silicone implant from the dissected tissue, samples were collected from the tissues on both dorsal and ventral sides to the prosthesis. The tissue samples were fixed in formalin solution and treated with paraffin. The paraffin-embedded tissue samples were sectioned at a thickness of $4 \mu \mathrm{m}$, and then prepared for histopathological examinations using hematoxylin and eosin (H\&E) staining.

The tissue sections were examined using the Olympus AX80 (Olympus Corporation, Tokyo, Japan) at a magnification of $100 \times$. The thickness of the capsule was measured after selection of the thickest and thinnest sites on the dorsal and ventral surfaces.

We measured the internal pressure of the prosthesis due to the formation of capsular contracture using a strain gauge transducer within a range of 0 to $10 \mathrm{mmHg}$ [15].

The number of blood vessels was measured by counting vessels within the visual field at a magnification of $200 \times ; 4$ random regions were selected from each tissue section and photographed. The number of blood vessels distributed in the interior and adjacent regions to the capsule was counted and then averaged.

\section{Statistical analysis}

Measurements of the thickness of the capsule and the number of blood vessels were expressed as median and range. Statistical analyses were performed using the SPSS version 22 (IBM Corp., Armonk, NY, USA), for which we used the Bonferroni method as a post hoc analysis. To minimize type 1 error, variables with an adjusted P-value of less than 0.05 were considered statistically significant. In addition, we performed a Spearman correlation analysis to identify the correlation between the thickness of the capsule and the number of blood vessels.

\section{RESULTS}

\section{Experimental animals}

Systemic conditions of the SD rats and surgical scar areas were monitored on a daily basis postoperatively. During the experiment, one SD rat from experimental group 1 and another from experimental group 2 died from unknown causes. There were no adverse effects associated with the laboratory procedures. The other SD rats remained healthy throughout the experiment. There were no adverse effects or complications in association with the VEGF treatment.

\section{Formation of the peri-implant capsule}

A fibrous capsule with a well-defined margin from the adjacent tissue surrounded the implant. On palpation, the capsule had a firm, smooth consistency (Fig. 2). There was no significant difference in the thickness of the capsule between the four groups on palpation.

On histopathological examinations, there was a fibrous capsule around the implant with a regular arrangement of tightly packed collagen fiber. The capsule was formed of an inner layer, surrounding the prosthesis, and an outer layer of loose connective tissue. The inner layer was filled with collagen fiber with a regular arrangement, accompanied by the presence of fibroblasts and a small number of lymphocytes. The outer layer was composed of collagen fiber, not in a regular arrangement (compared with the inner layer), and lower density. There were no significant differences in the type and

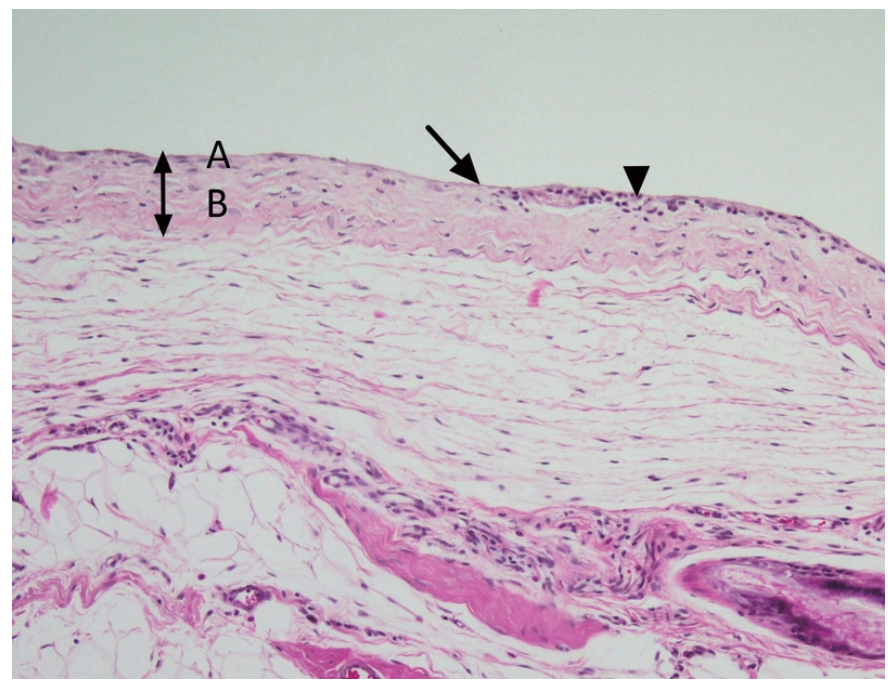

Fig. 3. Histopathological findings of the tissue sample. On histopathological examinations, the fibrous capsule around the prosthesis had a regular arrangement of dense collagen fiber. It was composed of an inner layer ( $A$ ) and an outer, loose connective tissue layer (B). The inner layer was filled with collagen fiber with a regular arrangement, accompanied by the presence of fibroblasts (long arrow) and a small number of lymphocytes (short arrow). The outer layer was composed of collagen fiber, not in a regular arrangement (compared with the inner layer), and lower density (hematoxylin and eosin [H\&E], x100). 

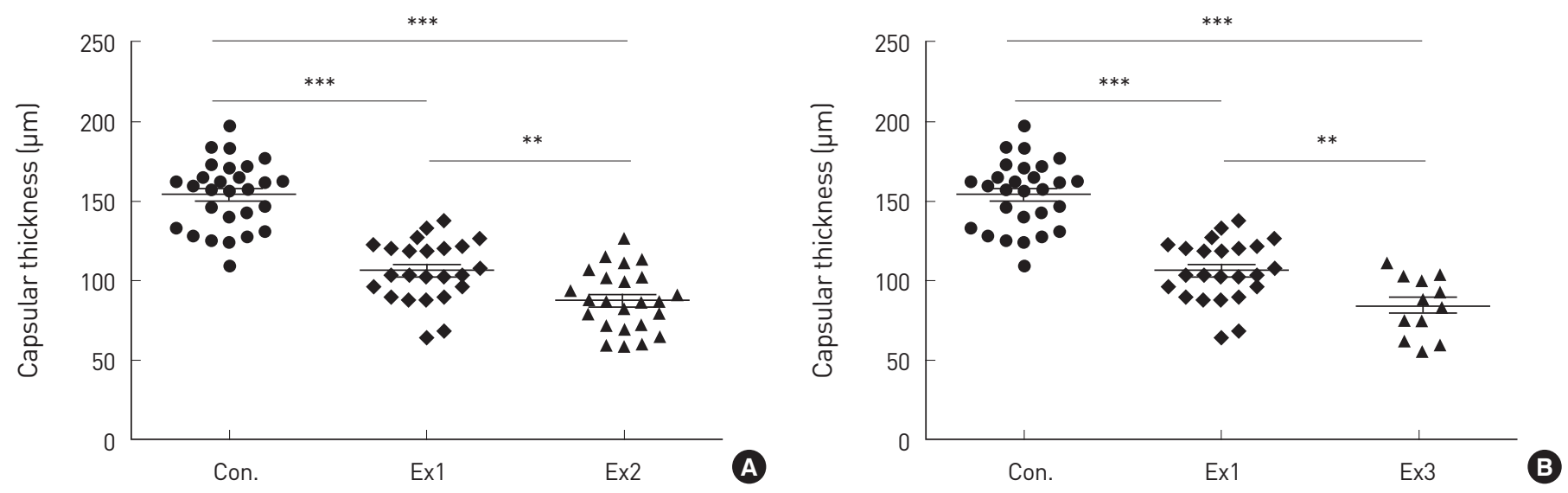

Fig. 4. (A, B) The thickness of the capsule. There was a significant difference in the thickness of the capsule between all experimental groups and the control group $(P<0.01)$. Statistical significance indicated with asterisks as follows: $* P<0.05, * * P<0.01$, and $* * * P<0.001$. Con, control group; Ex, experimental group.
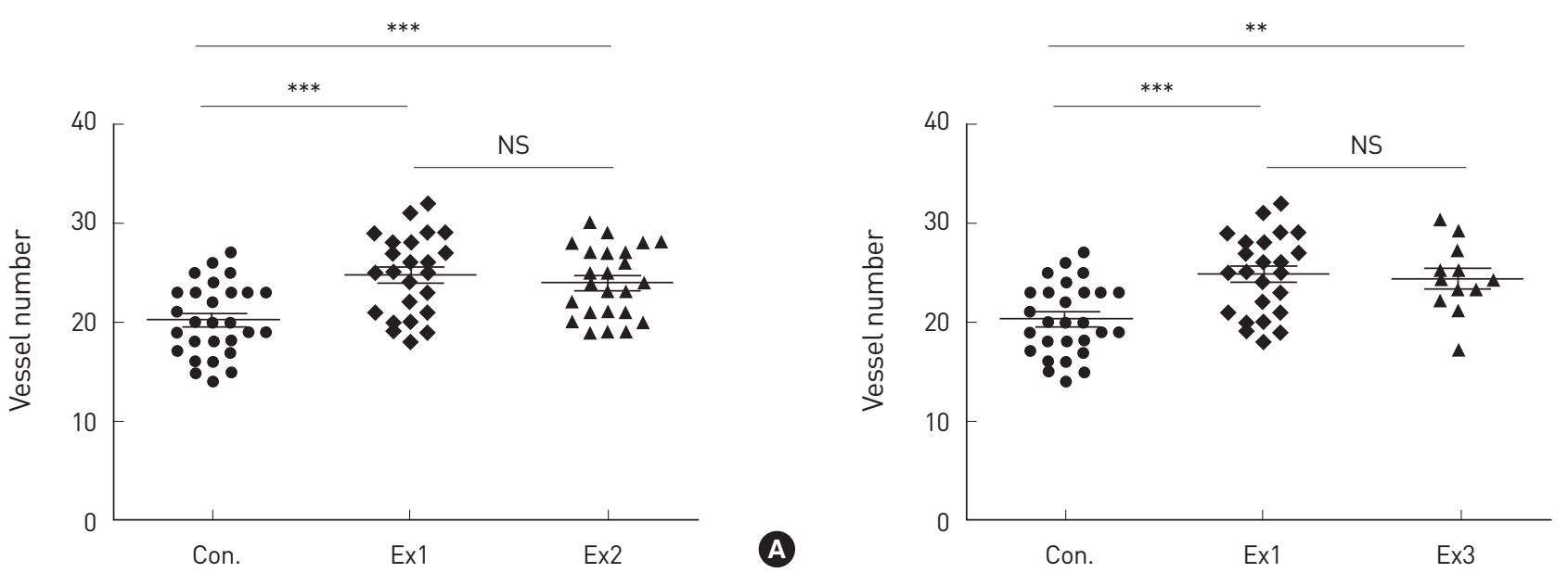

B

Fig. 5. (A, B) The number of blood vessels. The number of blood vessels formed around the capsule was significantly greater in all experimental groups compared with the control group. There was no significant difference between the experimental groups. Statistical significance indicated with asterisks as follows: ${ }^{*} \mathrm{P}<0.05,{ }^{* *} \mathrm{P}<0.01$, and ${ }^{* * * P}<0.001$. Con, control group; Ex, experimental group; NS, no significant.

number of cells between the experimental groups and the control group (Fig. 3).

The thickness of the capsule

The median thickness of the capsule around the prosthesis was $103.16 \mu \mathrm{m}$ (range, 64.13-137.42) in experimental group 1, 84.76 $\mu \mathrm{m}$ (range, $57.55-156.05$ ) in experimental group 2, $85.08 \mu \mathrm{m}$ (range, 54.75-110.66) in experimental group 3 and $158.08 \mu \mathrm{m}$ (range, 108.16197.13) in control group (Fig. 4). Post-hoc analysis showed that there was a significant difference in the thickness of the capsule between all the experimental groups and the control group $(\mathrm{P}<0.01)$. All the experimental groups had a significant difference in the thickness of the capsule compared to the control group $(\mathrm{P}<0.001)$. There was no significant difference between experimental group 2 and experimental group 3.
VEGF-induced angiogenesis

The median number of blood vessels formed around the capsule was 25 (range, 18-32) in experimental group 1, 24 (range, 19-30) in experimental group 2, 24 (range, 17-30) in experimental group 3, and 20 (range, 14-27) in the control group. The number of blood vessels formed around the capsule was significantly greater in all the experimental groups compared with the control group $(\mathrm{P}<0.05)$. There was no significant difference in it between the experimental groups (Fig. 5).

The correlation between the thickness of the capsule and the number of blood vessels

Spearman correlation analysis showed that there was a significant negative correlation between the thickness of the capsule and the number of blood vessels (Spearman correlation coefficient, 0.2732; 


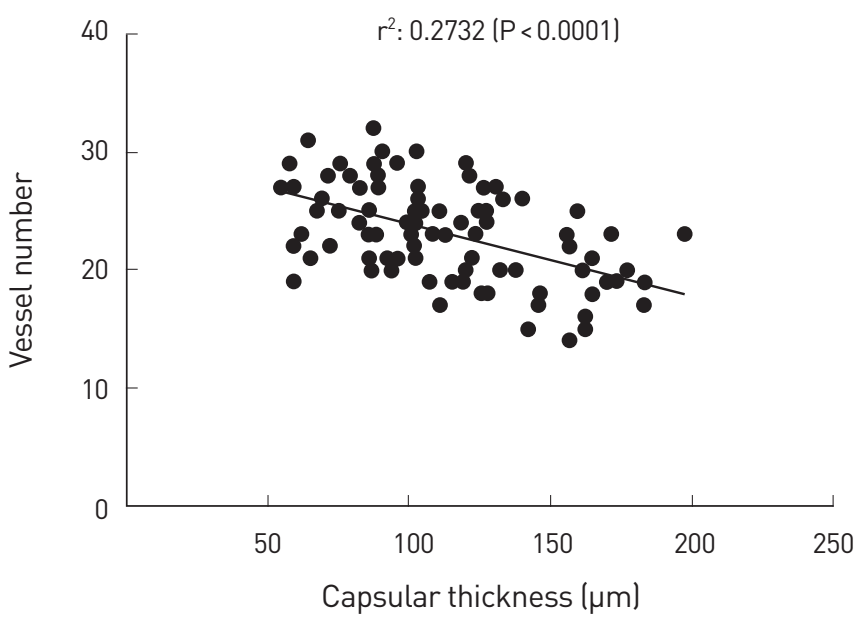

Fig. 6. The correlation between capsular contracture and vascularity. There was a significant negative correlation between the thickness of the capsule and the number of blood vessels (Spearman correlation coefficient, 0.2732; $P<0.0001$ ).

$\mathrm{P}<0.0001$ ) (Fig. 6).

\section{DISCUSSION}

One common complication of breast augmentation surgery, capsular contracture poses challenging problems for surgeons [5]. Studies have been conducted to examine the pathophysiology of capsular contracture, but this remains uncertain. In addition, there are no established treatment regimens for preventing occurrence. Previous studies suggested that tissue ischemia might be associated with the fibrosis $[12,13]$. Based on these previous publications, we hypothesized that inhibiting ischemic changes would be effective in reducing the occurrence of the capsular contracture, and we tested this hypothesis in the current animal experiment.

Our previous experiment showed that hESC-EPC CM was effective in promoting neo-angiogenesis and reducing the thickness of the capsule. VEGF is a major constituent of hESC-EPC CM, acting on the vascular endothelial cells and thereby promoting angiogenesis and increasing the vascular permeability in the peripheral tissue. VEGF has been reported to be effective in improving ischemic symptoms when administered at a lower dose [15-17]. We therefore designed the current experiment to examine the effects of a single administration of VEGF to improve ischemic symptoms.

Our results showed that all experimental groups showed a significant decrease in the thickness of the capsule as compared with the control group $(\mathrm{P}<0.01)$. This suggests that the VEGF might be effective in reducing the thickness of the capsule. Despite a lack of statistical significance, the thickness of the capsule was slightly smaller in experimental group 2 and experimental group 3 as compared with experimental group 1 . In addition, there was no significant difference between experimental group 2 and experimental group
3. We speculate that these effects might reach the highest level at a dose of 15 and $150 \mu \mathrm{g} / 0.1 \mathrm{cc}$.

On the other hand, the VEGF was initially administered at a consistent dose of $150 \mu \mathrm{g} / 0.1 \mathrm{cc}$ in experimental group 2 and experimental group 3. But it was additionally administered at a dose of $300 \mu \mathrm{g} / 0.2 \mathrm{cc}$ only in experimental group 3 on postoperative day 5 . Differences in the timing and methods of the administration of dosages might affect our results.

A dose-selection rationale is based on a previous published animal study about the effects of exogenous VEGF [18]. Thus, experimental group 1 was given VEGF at a dose that has been effective in the rat model. We obtained the results that were consistent with previous studies. Experimental group 1 was given a 10-fold lower dose than given to experimental group 2 and experimental group 3 . We expected that the toxic effect as well as the dose-dependent effectiveness of VEGF would occur in these groups, as VEGF may have angiogenic and tumorigenic effects $[19,20]$. VEGF is also associated with diseases such as rheumatoid arthritis, psoriasis, endometriosis, and Graves' disease [21]. Experimental group 1 did not show a significant difference in vascularity compared to experimental group 2 and experimental group 3 . We could not observe the toxic effects. We did assess the toxic effects of local administration of exogenous VEGF.

The number of blood vessels formed after treatment with VEGF was significantly larger in all experimental groups compared with the control group $(\mathrm{P}<0.05)$.

We failed to confirm the difference in the degree of infiltration of inflammatory cells between experimental groups and the control group. The formation of the capsular contracture was altered due to improvements in ischemic changes.

There was a significant negative correlation between the thickness of the capsule and the number of blood vessels (Spearman correlation coefficient, $0.2732 ; \mathrm{P}<0.0001$ ). These results support our hypothesis that administration of VEGF would promote angiogenesis and then reduce capsular contracture. It can therefore be inferred that VEGF-induced neo-angiogenesis would inhibit immune mechanisms underlying the fibrosis due to secretions of transforming growth factor-beta (TGF- $\beta$ ), hypoxia-inducible factor 1-alpha (HIF-1 $\alpha$ ), and angiotensin II during hypoxia [22]. Another study found that the expression of VEGF is not controlled by HIF-1a, but by protein kinase B/phosphatidylinositol 3-kinase (Akt/ $\mathrm{PI} 3 \mathrm{~K})$ and specificity protein 1 (SP1) pathways under hypoxia [23, 24]. This led to our speculation that there are no problems with VEGF-induced angiogenesis when administered in vivo.

The capsular thickness showed a significant difference between the control group and the experimental groups, and despite a lack of statistical significance between the experimental groups, it can be inferred that the effects of hESC-EPC CM in promoting angiogenesis and reducing the thickness of the capsule might originate from VEGF [14]. Further experimental studies are warranted to examine 
whether other constituents of hESC-EPC CM could have affected the thickness of the capsule and the number of blood vessels.

We found that experimental groups 1, 2, and 3 showed no significant difference in the number of blood vessels compared to the control group.

Our results indicate that ischemic changes might be involved in the formation of the capsular contracture. We attempted to obtain treatment effects by targeting the causes of the formation of the capsular contracture, which is a different feature from the pre-existing treatment modalities. Limitations of the current study are as follows: (1) The SD rats have a different immune system than humans. Moreover, a prosthesis is implanted in different sites in humans in an actual clinical setting. Further studies are needed to determine the optimal dose of VEGF in humans. (2) There were no shortterm toxic effects of VEGF in the current experiment. Further experimental studies are warranted to evaluate possible long-term toxic effects of VEGF. (3) There was insufficient evidence of vascularity on histopathology examination, for which cluster of differentiation 31 protein (CD31) immunohistochemistry will be needed.

We found that a single administration of VEGF reduced the thickness of the capsule and increased the vascularity around the implant, thus supporting the hypothesis that prevention of tissue ischemia can be another treatment strategy for capsular contracture. Further clinical studies are needed to determine effectiveness in prevention and treatment of capsular contracture.

\section{PATIENT CONSENT}

Patients provided written consent for the use of their images.

\section{REFERENCES}

1. Tamboto H, Vickery K, Deva AK. Subclinical (biofilm) infection causes capsular contracture in a porcine model following augmentation mammaplasty. Plast Reconstr Surg 2010;126:835-42.

2. Marques M, Brown SA, Oliveira I, et al. Long-term follow-up of breast capsule contracture rates in cosmetic and reconstructive cases. Plast Reconstr Surg 2010;126:769-78.

3. Barnsley GP, Sigurdson LJ, Barnsley SE. Textured surface breast implants in the prevention of capsular contracture among breast augmentation patients: a meta-analysis of randomized controlled trials. Plast Reconstr Surg 2006;117:2182-90.

4. Collis N, Coleman D, Foo IT, et al. Ten-year review of a prospective randomized controlled trial of textured versus smooth subglandular silicone gel breast implants. Plast Reconstr Surg 2000;106:786-91.

5. Handel N, Cordray T, Gutierrez J, et al. A long-term study of outcomes, complications, and patient satisfaction with breast implants. Plast Reconstr Surg 2006;117:757-67; discussion 68-72.

6. Henriksen TF, Fryzek JP, Hölmich LR, et al. Surgical intervention and capsular contracture after breast augmentation: a prospective study of risk factors. Ann Plast Surg 2005;54:343-51.

7. Spano A, Palmieri B, Taidelli TP, et al. Reduction of capsular thickness around silicone breast implants by zafirlukast in rats. Eur Surg Res 2008; 41:8-14.

8. Baker JL Jr. The effectiveness of alpha-tocopherol (vitamin E) in reducing the incidence of spherical contracture around breast implants. Plast Reconstr Surg 1981;68:696-9.

9. Kang Y, Lee DA, Higginbotham EJ. In vitro evaluation of antiproliferative potential of calcium channel blockers in human Tenon's fibroblasts. Exp Eye Res 1997;64:913-25.

10. Zimman OA, Toblli J, Stella I, et al. The effects of angiotensin-converting-enzyme inhibitors on the fibrous envelope around mammary implants. Plast Reconstr Surg 2007;120:2025-33.

11. Huang CK, Handel N. Effects of Singulair (montelukast) treatment for capsular contracture. Aesthet Surg J 2010;30:404-8.

12. Higgins DF, Kimura K, Bernhardt WM, et al. Hypoxia promotes fibrogenesis in vivo via HIF-1 stimulation of epithelial-to-mesenchymal transition. J Clin Invest 2007;117:3810-20.

13. Norman JT, Clark IM, Garcia PL. Hypoxia promotes fibrogenesis in human renal fibroblasts. Kidney Int 2000;58:2351-66.

14. Kim $\mathrm{CH}$, Kim DH, Oh SH, et al. Human embryonic stem cell-derived endothelial precursor cell conditioned medium reduces the thickness of the capsule around silicone implants in rats. Ann Plast Surg 2015; 75:348-52.

15. Bastos ÉM, Neto MS, Ferreira LM, et al. Experimental model of capsular contracture in silicone implants. Acta Cir Bras 2003;18:22-8.

16. Pang Y, Lineaweaver WC, Lei MP, et al. Evaluation of the mechanism of vascular endothelial growth factor improvement of ischemic flap survival in rats. Plast Reconstr Surg 2003;112:556-64.

17. Zhang F, Oswald TM, Lin L, et al. Improvement of full-thickness skin graft survival by application of vascular endothelial growth factor in rats. Ann Plast Surg 2008;60:589-93.

18. Seify H, Bilkay U, Jones G. Improvement of TRAM flap viability using human VEGF-induced angiogenesis: a comparative study of delay techniques. Plast Reconstr Surg 2003;112:1032-9.

19. Shen P, Jiao Z, Zheng JS, et al. Injecting vascular endothelial growth factor into the temporomandibular joint induces osteoarthritis in mice. Sci Rep 2015;5:16244.

20. Ferrara N, Gerber HP, LeCouter J. The biology of VEGF and its receptors. Nat Med 2003;9:669-76.

21. Takahashi $\mathrm{H}$, Shibuya M. The vascular endothelial growth factor (VEGF)/ VEGF receptor system and its role under physiological and pathological conditions. Clin Sci (Lond) 2005;109:227-41.

22. Ferrara N, Davis-Smyth T. The biology of vascular endothelial growth factor. Endocr Rev 1997;18:4-25.

23. Krock BL, Skuli N, Simon MC. Hypoxia-induced angiogenesis: good and evil. Genes Cancer 2011;2:1117-33.

24. Trisciuoglio D, Iervolino A, Zupi G, et al. Involvement of PI3K and MAPK signaling in bcl-2-induced vascular endothelial growth factor expression in melanoma cells. Mol Biol Cell 2005;16:4153-62. 